\title{
Symptom Severity Related With Contraction Peaks in Patients With Jackhammer Esophagus
}

\author{
Yinglian Xiao, ${ }^{1 *}$ Dustin A Carlson, ${ }^{2}$ and John E Pandolfino ${ }^{2}$ \\ ${ }^{I}$ Department of Gastroenterology and Hepatology, the First Affiliated Hospital, Sun Yat-sen University, Guangzhou, China; and ${ }^{2}$ Department of \\ Medicine, the Feinberg School of Medicine, Northwestern University, Chicago, IL, USA
}

\section{Background/Aims}

Jackhammer esophagus is an uncommon heterogeneous motility disorder associated with a distal contractile integral $>8000$ $\mathrm{mmHg} \cdot \mathrm{sec} \cdot \mathrm{cm}$. The spectrum of abnormality ranges from a relatively normal looking contraction to chaotic repetitive contractions akin to a jackhammer. Although previous studies have shown an uncertain correlation between peristaltic vigor and symptoms, we hypothesize that symptoms may be more severe with repetitive contractions as opposed to an elevated contractile measure. Thus, this study aims to investigate whether symptom severity is related to the contraction pattern in the patients with hypercontractile esophagus.

\section{Methods}

Patients with hypercontractile esophagus were retrospectively identified, their demographic and high-resolution manometry characteristics were collected. Contraction pattern on high-resolution manometry was categorized into single-peak and multiple-peak. Comparison was performed between patients with single-peak and multiple-peak.

\section{Results}

Altogether 35 patients (age range, 45-70 years; female:male, 24:11) were included. Seven patients presented with single-peak hypercontractile swallows, while 28 patients presented with multiple-peak hypercontractile swallows. The patients with multiple-peak showed higher Brief Esophageal Dysphagia Questionnaire scores compared with patients with single-peak. The jackhammer swallows with multiple-peak were associated with higher distal contractile integral values, longer distal latency intervals, and a lower integrated relaxation pressure.

\section{Conclusions}

Repetitive contractions akin to a jackhammer were common amongst patients with hypercontractile esophagus. Patients with the jackhammer pattern also presented with more severe symptoms. Further distinction of hypercontractile esophagus into a jackhammer dominant subtype may be warranted.

(J Neurogastroenterol Motil 2021;27:540-544)

\section{Key Words}

Deglutition disorders; Peristalsis; Swallows

Received: October 8, 2020 Revised: March 5, 2021 Accepted: April 23, 2021

(a) This is an Open Access article distributed under the terms of the Creative Commons Attribution Non-Commercial License (http://creativecommons. org/licenses/by-nc/4.0) which permits unrestricted non-commercial use, distribution, and reproduction in any medium, provided the original work is properly cited.

*Correspondence: Yinglian Xiao, MD, PhD Department of Gastroenterology and Hepatology, the First Affiliated Hospital, Sun Yat-sen University, 58 Zhongshan Road 2, Guangzhou 510080, China Tel: +86-20-87755766 (ext. 8182), Fax: +86-20-87332916, E-mail: xyingl@mail.sysu.edu.cn 


\section{Introduction}

The Chicago classification version 3.0 (v3.0) has defined jackhammer esophagus or hypercontractile esophagus as a motility disorder characterized by more than 2 hypercontractile swallows (distal contractile integral [DCI] $>8000 \mathrm{mmHg} \cdot \mathrm{sec} \cdot \mathrm{cm}$ ) among the total 10 supine liquid swallows. ${ }^{1}$ Although former studies have shown that dysphagia and chest pain are the major symptoms related to hypercontractile esophageal motor disorders, there is an uncertain correlation between contractile activity and symptoms noted on both conventional and high-resolution manometry (HRM). ${ }^{2,3}$ Thus, it has been hypothesized that the pain and dysphagia seen with hypercontractile esophagus are not directly related to the contractile vigor, but may be related to subtle obstruction or local ischemia due to microvascular compression. ${ }^{4}$ Also, clinical observations of hypercontractility in patients with eosinophilic esophagitis or gastroesophageal reflux disease has been reported. ${ }^{5,6}$ Thus, further studies focusing on a more detailed description of the contractile pattern in jackhammer esophagus are needed to determine whether these differences have relevance beyond the DCI value.

Recently, we tried a new measurement tool, where the up- and down-slope of the peristaltic wave were separated in order to make a more detailed measurement for both the contraction and relaxation phases of contraction. ${ }^{7}$ Using this new algorithm, we found that the abnormalities in contractile integral of the post-peak phase are more significant in jackhammer esophagus patients with higher dysphagia scores. ${ }^{8}$ This approach may have masked the abnormalities in the swallows with persistent repetitive contraction peaks since only the first peak was analyzed in this new approach. However, we did note that there was at least some anecdotal suggestion that the pa- tients with higher post-peak contractile integrals were more likely to have the repetitive jackhammer pattern. Given these observations, we sought to determine whether repetitive contractions alone could be associated with dysphagia severity in patients with a hypercontractile esophageal disorder.

\section{Materials and Methods}

\section{Study Subjects}

Jackhammer esophagus patients, who were aged 18-85 and had HRM in the Esophageal Center at Northwestern Motility Lab due to esophageal symptoms were retrospectively reviewed. Those patients between August 2008 and August 2015 who completed the Brief Esophageal Dysphagia Questionnaire (BEDQ) and had more than 2 supine swallows with DCI $>8000 \mathrm{mmHg} \cdot \mathrm{sec} \cdot \mathrm{cm}$ during HRM were retrospectively included. Patients with previous upper gastrointestinal tract surgery were excluded. The BEDQ was previously termed the impaction dysphagia questionnaire and was updated after the validation study by Taft et $\mathrm{al}^{9}$ in 2016 in our lab. The HRM and BEDQ of these included patients was performed in the same day. These subjects have been previously described. ${ }^{8}$ The current research protocol was ratified by the Northwestern University Institutional Review Board (IRB No. NU IRB STU00011084).

\section{High-resolution Manometry}

HRM procedure was performed as previously described. ${ }^{7}$ The esophageal pressure topography was analyzed using the Manoview analysis software incorporated by the manufacturer (Medtronic Inc. Minneapolis, MN, USA). The DCI, distal latency (DL) as well as

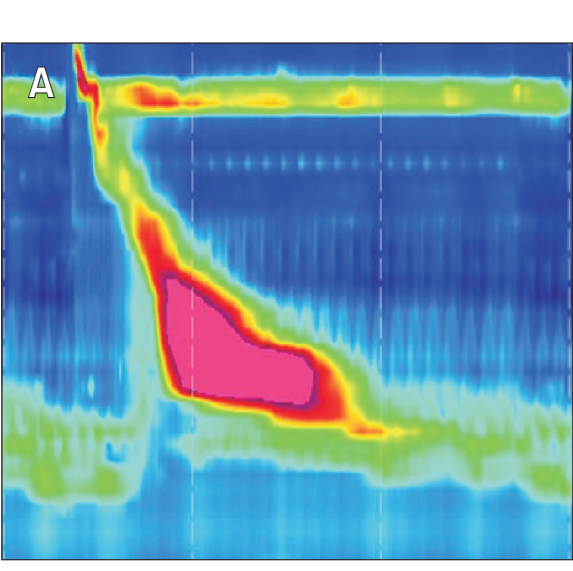

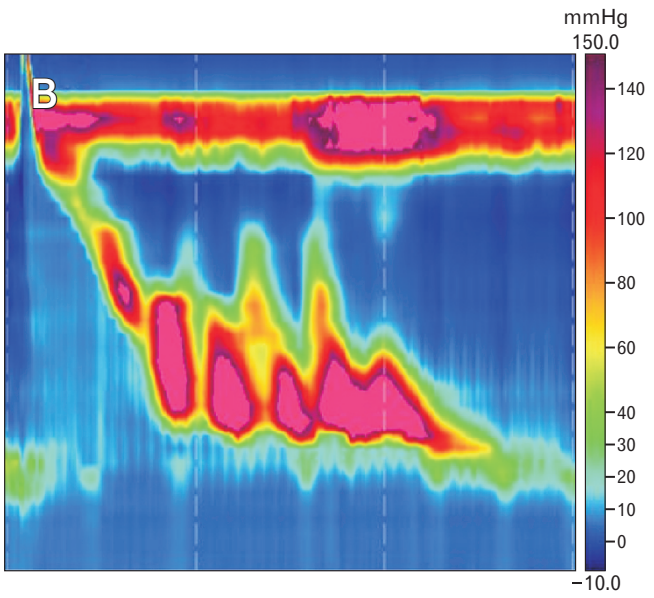

Figure. Demonstration of jackhammer swallows with single-peak (A) and multiplepeak (B). 
integrated relaxation pressure (IRP) of the supine liquid swallows were analyzed among all the jackhammer patients.

Those swallows with DCI $>8000 \mathrm{mmHg} \cdot \mathrm{sec} \cdot \mathrm{cm}$ were recognized as jackhammer swallows, and these swallows from each patient were selected to compare the parameters between single-peak swallow and multiple-peak swallow. The definition of multiple-peak swallow was described in the study by Clouse et $\mathrm{al}^{10}$ : there would be 2 peaks at least with the least interval of 1 second between pressure peaks, the amplitude of the peak pressure should be at least 10 $\mathrm{mmHg}$ greater than the trough between 2 peaks with the trough pressure $>0$. If the patients had at least 1 episode of jackhammer swallow with multiple-peak, they would be categorized into multiple-peak group, otherwise single-peak group (Figure).

\section{Statistical Methods}

All the supine swallows and the jackhammer swallows from each subjects were analyzed. Data were reported as median (95\% confidence interval). The Wilcoxon-signed rank test was used to find out statistical significance. Pearson correlation was performed to find out the correlation between the BEDQ score and the peak numbers. The significance level was set at 0.05 .

\section{Results}

A total of 35 patients (age 45-70 years, female:male [24:11]) out of 2074 patients for esophageal function testing were included. Twenty-one patients were with dysphagia, 11 with reflux, and 3 with chest pain. The medication of these patients included calcium channel blockers ( $\mathrm{n}=5)$, opiates ( $\mathrm{n}=5)$, neuromodulators including venlafaxine, escitalopram, and imipramine $(\mathrm{n}=5)$, and none of them were on nitrates. There were 24 patients who were on proton

Table 1. The Comparison Between Jackhammer Patients With Single-peak and Multiple-peak

\begin{tabular}{lccc}
\hline \multicolumn{1}{c}{ Item } & $\begin{array}{c}\text { Single-peak group } \\
(\mathrm{n}=7)\end{array}$ & $\begin{array}{c}\text { Multiple-peak } \\
\text { group }(\mathrm{n}=28)\end{array}$ & $P$-value \\
\hline Age (yr) & $65(52-66)$ & $59(49-68)$ & 0.505 \\
Gender (M/F) & $3: 4$ & $8: 20$ & 0.466 \\
DCI (mmHg.sec.cm) & $6191(5820-7470)$ & $9345(6460-14252)$ & 0.025 \\
IRP (mmHg) & $9.1(8.2-13.9)$ & $9.6(6.5-13.0)$ & 0.135 \\
DL (sec) & $6.2(5.2-7.4)$ & $7.5(5.8-9.3)$ & 0.825 \\
BEDQ & $5(0-10)$ & $14(7-22)$ & 0.020 \\
\hline
\end{tabular}

M, male; F, female; DCI, distal contractile integral; IRP, integrated relaxation pressure; DL, distal latency; BEDQ, Brief Esophageal Dysphagia Questionnaire.

Data are presented as median $(95 \% \mathrm{CI})$. pump inhibitors, but only 11 patients had classic reflux symptoms. On upper endoscopy, there was only 1 patient with esophagitis, and 2 patients with subtle small caliber in the distal esophagus while no eosinophilic infiltration were found after biopsy, another 2 patients with a hiatal hernia $>3 \mathrm{~cm}$.

Among all the patients, 7 patients presented as single-peak jackhammer swallows, while 28 patients presented as multiplepeak jackhammer swallows. The comparison between these patients with single-peak and multiple-peak is listed in Table 1. The IRP and DL were similar between these patients with single-peak and multiple-peak, while the patients with multiple-peak presented with higher DCI compared with patients with single-peak patterns. $\mathrm{Pa}-$ tients with multiple-peak had higher BEDQ scores compared with patients with single-peak. However, there was no correlation between the BEDQ score and the peak numbers among the patients with jackhammer esophagus.

The jackhammer swallows from all the included patients were selected for further analysis. Altogether, there were 193 hypercontractile swallows, 71 swallows with single-peak patterns while the other 122 swallows had multiple-peak. The comparison between the single-peak hypercontractile swallows and multiple-peak hypercontractile swallows are listed in Table 2. The Jackhammer swallows with multiple-peak were noted to have higher DCI values and longer DL intervals. Additionally, the swallows with the multiplepeak Jackhammer pattern had a significantly lower median IRP.

\section{Discussion}

Although considered to be a major motor disorder, jackhammer esophagus has been a confusing entity primarily due to the heterogeneity of its contractile pattern and its clinical presentation. Using the definition of 2 or more swallows with a DCI $>8000$ does not adequately describe the spectrum of motility patterns seen in this manometric classification and thus, we sought to determine

Table 2. The Comparison of Jackhammer Swallows With Singlepeak and Multiple-peak

\begin{tabular}{lccr}
\hline \multicolumn{1}{c}{ Item } & $\begin{array}{c}\text { Single-peak } \\
\text { swallow }(\mathrm{n}=71)\end{array}$ & $\begin{array}{c}\text { Multiple-peak } \\
\text { swallow (n=122) }\end{array}$ & $P$-value \\
\hline $\begin{array}{l}\text { DCI } \\
(\mathrm{mmHg} \cdot \mathrm{sec} \cdot \mathrm{cm})\end{array}$ & $9946(8712-13$ 142) & $13914(10$ 104-20 583) & 0.008 \\
IRP (mmHg) & $10.7(7.6-14.1)$ & $7.9(5.1-13.9)$ & $<0.001$ \\
DL (sec) & $7.0(5.9-8.2)$ & $7.7(6.8-9.2)$ & 0.007 \\
\hline $\begin{array}{l}\text { DCI, distal contractile integral; IRP, integrated relaxation pressure; DL, distal } \\
\text { latency. }\end{array}$ & & \\
Data are presented as median (95\% CI).
\end{tabular}


whether repetitive contractions had additional value to subtyping this group of patients. Our results support that the repetitive contraction pattern was commonly seen in patients with 2 or more swallows with a DCI $>8000$ and that this pattern was associated with higher symptom severity. Thus, it appears that the repetitive jackhammer pattern is an important subtype of hypercontractile esophageal disorders and the nomenclature should be revised to reflect the heterogeneity of hypercontractile disorders.

The heterogeneity of hypercontractile esophagus has been reported. Agrawal et al ${ }^{2}$ reviewed a group of 56 patients with nutcracker esophagus using conventional manometry, and they found that patients with contraction amplitudes greater than $260 \mathrm{mmHg}$ on manometry had a more homogeneous clinical presentation, whereas patients with distal esophageal contraction amplitudes between $180 \mathrm{mmHg}$ and $220 \mathrm{mmHg}$ had a more heterogeneous presentation. Our study is also not the first to focus on repetitive contractions as an important variant of the hypercontractile disorders. Clouse et $\mathrm{al}^{10}$ examined 74 double-peaked waves detected in subjects with unexplained symptoms using the topographic analysis. Through their analysis they noted that the second peak in a doublepeak wave is typically a short, simultaneous, or retrograde pressure event in the distal esophageal body. This indicated that there was chaotic contraction originating from the distal esophagus in the double-peak swallow which may distinguish itself from those with single-peak swallow.

Additionally, our group recently focused on assessing the morphology of the contractile wave by separating the contraction into a pre- and post-peak contraction to better define abnormalities associated with return of the muscle to its baseline resting state. We also looked at the timing of the peak propagation to determine whether a lack of intra-contractile synchrony was associated with worse outcomes. These results suggest that symptom severity was associated to a longer duration of the post-peak activity during the esophageal contraction. ${ }^{8}$ Further, this study has also confirmed that chaotic contraction patterns were also more common in symptomatic patients with hypercontractile esophagus. ${ }^{11}$

A recent study in Europe identified 34 patients with Jackhammer esophagus ${ }^{12}$ and showed that dysphagia was associated with DCI and an elevated intrabolus pressure. However, they found that the differences in HRM and clinical characteristics between subgroups based on the contraction type (single- or multi-peaked) were limited. A larger cross-sectional study from France reported on a 227 patient cohort with Jackhammer esophagus. ${ }^{13}$ This study found that none of the individual symptoms were significantly associated with any of the manometric parameters defined, except for dyspha- gia (associated with the mean of all DCIs $>8000 \mathrm{mmHg} \cdot \mathrm{sec} \cdot \mathrm{cm}$ ). Instead of using a single symptom to find the relationship between the manometric features and symptom severity, our study used the BEDQ to evaluate the clinical symptom profile of these patients, and found a distinct relationship between multiple-peak patterns and symptom score. Thus, our study focused on a more granular level assessment of the contractile morphology.

The definition of Jackhammer esophagus has evolved from 1 swallow with DCI $>8000$ to 2 swallows since the Chicago classification v3.0. The pattern of contraction has no role in the diagnosis criteria, while multiple-peak contractions has been found in about $80 \%$ of all the Jackhammer esophagus. ${ }^{14}$ In our current study, the multiple-peak pattern was associated with a higher BEDQ and thus, the multiple-peak pattern may potentially help better refine the diagnosis of jackhammer esophagus.

Another interesting finding in this study was related to the IRP values. The IRP was significantly higher in the swallows with single-peak, although still within the normal range. The comparison between the individual single-peak and multiple-peak hypercontractile swallows showed higher IRP values and more obstruction to flow with the single-peak swallows. There have been reports of an overlap of jackhammer patterns with type III achalasia and there are also case reports of Jackhammer esophagus progressing to achalasia. ${ }^{15,16}$ These patients may represent a different category of hypercontractile disorders more akin to spastic achalasia and the vigorous contractions may be related to a more forceful attempt to push the bolus through an obstruction.

There were also some limitations in our research. The sample size was relatively small, only 7 patients with single-peak were identified. Jackhammer esophagus is not a common motility disorder and a multicenter study could be helpful to determine whether these findings are generalizable. Additionally, the patient population was heterogeneous in terms of presentation. However, this is the nature of hypercontractile disorders as they can be seen in patients with gastroesophageal reflux disease or a frank overt mechanical obstruction at the esophagogastric junction.

During the initial descriptions of jackhammer esophagus, the pattern of repetitive contractions was the most distinguishing component that separated this disorder from nutcracker esophagus. A nutcracker typically generates a single long contraction on a walnut to crack the shell while jackhammers have repetitive strong bursts that penetrate rock and concrete. This distinction has been lost in the Chicago classification v3.0 definition and it appears that a more logical classification would label all patients with a DCI $>8000$ as having a hypercontractile esophagus and allowing for a distinc- 
tion to call a single-peak pattern "nutcracker esophagus" and the multiple-peak pattern "jackhammer esophagus." Whether this new classification or rearrangement will provide a better scheme to define these disorders will require larger sample size studies and an assessment of outcome using carefully designed clinical intervention trials with peroral endoscopic myotomy or medications.

In conclusion, our study supports that the repetitive jackhammer pattern is a more severe variant within the hypercontractile classification defined using a DCI $>8000$.

\section{Financial support: None.}

Conflicts of interest: John E Pandolfino: consulting and educational (Metronic) and consulting (Sandhill Scientific Inc). Dustin A Carlson: speaking and consulting (Medtronic). No other conflicts for remaining author.

Author contributions: Yinglian Xiao: study concept and design, acquisition of data, analysis and interpretation of data, and drafting and finalizing the manuscript; Dustin A Carlson: study concept and design and finalizing the manuscript; and John E Pandolfino: study concept and design, finalizing the manuscript, and guarantee of the study.

\section{References}

1. Kahrilas PJ, Bredenoord AJ, Fox M, et al. The Chicago classification of esophageal motility disorders, v3.0. Neurogastroenterol Motil 2015;27:160-174.

2. Agrawal A, Hila A, Tutuian R, Mainie I, Castell DO. Clinical relevance of the nutcracker esophagus: suggested revision of criteria for diagnosis. J Clin Gastroenterol 2006;40:504-509.

3. Richter JE, Bradley LA, Castell DO. Esophageal chest pain: current controversies in pathogenesis, diagnosis, and therapy. Ann Intern Med 1989;110:66-78.
4. Balaban DH, Yamamoto Y, Liu J, et al. Sustained esophageal contraction: a marker of esophageal chest pain identified by intraluminal ultrasonography. Gastroenterology 1999;116:29-37.

5. Melchior C, Chiavelli H, Leroi AM, Ducrotté P, Gourcerol G. Recovery of a "jackhammer esophagus" after the treatment of an eosinophilic esophagitis. Am J Gastroenterol 2012;107:952-954.

6. Kristo I, Schwameis K, Maschke S, et al. Phenotypes of jackhammer esophagus in patients with typical symptoms of gastroesophageal reflux disease responsive to proton pump inhibitors. Sci Rep 2018;8:9949.

7. Xiao Y, Carlson DA, Lin Z, Rinella N, Sifrim D, Pandolfino JE. Assessing the pre- and postpeak phases in a swallow using esophageal pressure topography. Neurogastroenterol Motil 2017;29:e13099.

8. Xiao Y, Carlson DA, Lin Z, Alhalel N, Pandolfino JE. Jackhammer esophagus: assessing the balance between prepeak and postpeak contractile integral. Neurogastroenterol Motil 2018;30:e13262.

9. Taft TH, Riehl M, Sodikoff JB, et al. Development and validation of the brief esophageal dysphagia questionnaire. Neurogastroenterol Motil 2016;28:1854-1860.

10. Clouse RE, Staiano A, Alrakawi A. Topographic analysis of esophageal double-peaked waves. Gastroenterology 2000;118:469-476.

11. Xiao Y, Carlson DA, Lin Z, Pandolfino JE. Chaotic peak propagation in patients with jackhammer esophagus. Neurogastroenterol Motil 2020;32:e13725.

12. Herregods TV, Smout AJ, Ooi JL, Sifrim D, Bredenoord AJ. Jackhammer esophagus: observations on a European cohort. Neurogastroenterol Motil 2017;29:e12975.

13. Philonenko S, Roman S, Zerbib F, et al. Jackhammer esophagus: clinical presentation, manometric diagnosis, and therapeutic results-results from a multicenter French cohort. Neurogastroenterol Motil 2020;32:e13918.

14. Roman S, Pandolfino JE, Chen J, Boris L, Luger D, Kahrilas PJ. Phenotypes and clinical context of hypercontractility in high-resolution esophageal pressure topography (EPT). Am J Gastroenterol 2012;107:37-45.

15. Quader F, Mauro A, Savarino E, et al. Jackhammer esophagus with and without esophagogastric junction outflow obstruction demonstrates altered neural control resembling type 3 achalasia. Neurogastroenterol Motil 2019;31:e13678.

16. Huang L, Rezaie A. Progression of jackhammer esophagus to achalasia. J Neurogastroenterol Motil 2016;22:348-349. 